\title{
Fraud detection in telecommunication industry using Gaussian mixed model
}

\begin{abstract}
The prevalence of fraud activities in telecommunication industry has reached a critical point so that efficient algorithms to identify such activities are greatly needed. In this article, we propose a new fraud detection algorithm using Gaussian mixed model (GMM), a probabilistic model successfully used in speech recognition problem. The expectation maximization algorithm is used to estimate the parameter of the model such that the initial values of the algorithm is determined using the kernel method. Using data obtained from one of the leading telecommunication companies in Malaysia, we show that the proposed algorithm has successfully not only detected fraud calls as suspected by the company, but also to identify suspicious calls which can be candidates of fraud call. The proposed algorithm is easy to implement with a great potential to be extended to detect (billed/outgoing) fraud calls and hence reduces the lost incurred by the telecommunication companies.
\end{abstract}

Keyword: Fraud; Telecommunication; Gaussian mixed models; EM algorithm; Kernel method 